\title{
Age of Acquisition and Spoken Words: Examining Hemispheric Differences in Lexical Processing
}

\section{Julio González-Alvarez ${ }^{\text {[GQ: } 1]}$}

Department of Basic, Clinical, and Biological Psychology, University Jaume I, Spain

\section{Teresa Cervera-Crespo}

Department of Basic Psychology, University of Valencia, Spain

\begin{abstract}
The relationship between the age of acquisition ( $A \circ A)$ of words and their cerebral hemispheric representation is controversial because the experimental results have been contradictory. However, most of the lexical processing experiments were performed with stimuli consisting of written words. If we want to compare the processing of words learned very early in infancywhen children cannot read-with words learned later, it seems more logical to employ spoken words as experimental stimuli. This study, based on the auditory lexical decision task, used spoken words that were classified according to an objective criterion of AoA with extremely distant means ( 2.88 vs. 9.28 years old). As revealed by the reaction times, both early and late words were processed more efficiently in the left hemisphere, with no AoA $\times$ Hemisphere interaction. The results are discussed from a theoretical point of view, considering that all the experiments were conducted using adult participants.
\end{abstract}

\section{Keywords}

Age of acquisition, spoken words, hemispheric differences, auditory lexical decision task

The age of acquisition (AoA), or when particular words are acquired in life, is a significant variable that, according to scientific research, exerts a durable influence upon lexical processing. The effects of AoA have been consistently reported for a variety of tasks: picture naming, word reading, word recognition, or lexical decision tasks (Cortese \& Khanna, 2007; see Johnston \& Barry, 2006, for a review). Furthermore, words learned early in life are more resilient to brain damage in aphasia patients than words learned later in life (Brysbaert \& Ellis, 2016). 
One suggested explanation for the AoA effects is that the early acquired words have some advantage owing to their bilateral representation in the brain. In contrast, late acquired words would be processed mainly in the dominant hemisphere responsible for speech, which is usually the left hemisphere in most people. However, the relationship between the AoA of words and hemispheric differences in lexical processing remains controversial.

In an influential chapter, Gazzaniga (1974) noted that the corpus callosum undergoes great growth in humans during the first 4 years of life, and the myelinization of this anatomical structure is not completed until several years later. According to this author, before the full development and myelinization of the corpus callosum, both cerebral hemispheres cannot fully communicate with each other - they function in parallel as if they were a split brain. Consequently, the words learned early in childhood would have a bilateral representation in the child's (and adult's) brain. However, to the extent that the corpus callosum is fully developed and myelinates, and simultaneously the left hemisphere becomes the dominant hemisphere for language, lexical processing is lateralized. Consequently, the words learned later in childhood would have an asymmetrical representation mainly focused on the left hemisphere.

Ellis and Young (1977) conducted the first empirical test of this hypothesis using a divided visual field paradigm. Printed stimulus words were presented tachistoscopically left or right of a central fixation point to be recognized by the participants. The words were nouns acquired early versus later in life. However, the results did not confirm the hypothesis, and the expected interaction between the AoA and the visual field (cerebral hemisphere) was not found. They observed a superiority of the right visual field (left hemisphere) over the left visual field (right hemisphere) in the same degree of intensity for both words acquired early and words acquired later in life - that is, compared with later words, early words did not show a greater degree of hemispheric bilaterality (less asymmetry).

Using a bilateral presentation of stimuli, Young and Bion (1980) obtained the same results. In each trial, a different word was simultaneously presented in each visual hemifield, and the participants were required to recognize both words. Two different times of exposure- 30 and $150 \mathrm{~ms}-$ were used, and in both conditions, the data analysis revealed an overall superiority of the right visual field (left hemisphere) for both early and later words, without any interaction between AoA and visual field. Young et al. (1982) obtained similar results regarding the age of reading acquisition. They introduced this parameter because the stimuli used in the divided visual paradigms were always printed words. The age of reading acquisition, they found, did not affect hemispheric asymmetries for naming nouns.

Previous experiments employed words that had been rated retrospectively for AoA by adults, but Boles et al. (1982) used stimuli selected from a corpus of words actually observed in the speech of very young children (under 2 years) studied by Katherine Nelson (1973). Compared with later-acquired words, the authors did not find differences in the right-visual-field superiority in a divided visual field paradigm. Again, an analysis of variance yielded significant main effects of the visual field (better word recognition in the right field) and AoA (better performance for early acquired words); but importantly, there was no interaction between the AoA and the visual field. Using the same experimental paradigm, Beaton et al. (2007) found an overall advantage of the left hemisphere (right visual field) for early and later-learned words by monolingual English speakers, but the laterality index was not different for both types of words. Also studying fluent bilingual English-Welsh speakers, the same authors observed that the magnitude of the laterality index was unaffected by the AoA (before or after 5-6years old) of the second language.

However, a more recent study reported a different pattern of results. Bowers et al. (2013) noted that none of the prior studies had measured reaction times (RTs) during lexical 
processing (only the accuracy of word recognition). RTs play a crucial role in experimental cognitive psychology because they are a direct reflection of the ongoing processes. These authors performed two experiments based on a lexical decision task within a divided visual field paradigm, taking the RT as the main dependent variable. In the first experiment, 160 items ( 80 words and 80 nonwords) were administered. The early acquired words corresponded to an AoA of 3-4 years old on a normative rating study (Gilhooly \& Logie, 1980). The lateracquired words corresponded to an AoA of 7-8 years old on the same normative rating study. Furthermore, both types of words were matched on several psycholinguistic variables, including word length, number of syllables, lexical frequency, imageability, familiarity, and orthographic neighborhood size. The analysis of the RTs of correct responses showed a significant main effect of the AoA field (superiority of early acquired words), but it also yielded a significant interaction between the AoA and the visual field. The main effect of the visual field was not significant. Curiously, for early acquired words, the participants responded faster when the stimulus was presented in the left visual field (right hemisphere) than the right (left hemisphere). For late-acquired words, as expected, the participants responded faster when the stimulus was presented in the right visual field (left hemisphere) than the left (right hemisphere). A second experiment with another set of stimuli replicated the results. Their results, the authors stated, were consistent with the idea that the right hemisphere may play an important role in the first years of life.

Now, the stimuli used in all the above-mentioned studies were printed words. When we want to compare the processing of very early learned words (under 3-4years old) versus later-learned words, it seems more logical to use spoken words as experimental stimuli, because such words are learned aurally (not read) by young children.

This study has the following characteristics. First, the stimuli were spoken words (AoA $M: 2.9$ vs. 9.3 years old), matched on several relevant psycholinguistic variables. Second, the AoA was an objective parameter directly obtained from a pool of more than 700 children rather than being derived from retrospective ratings by adults, subject to memory biases or stereotypes. Third, the main dependent variable was the RT in an auditory lexical task, and the response accuracy was the secondary dependent variable. And fourth, a large sample of participants (over a hundred) was used to ensure sufficient power of analysis.

\section{Method}

\section{I.I Participants}

Participants were 111 adults of both sexes (90 females) whose age range was 19-43 years $(M=20.97 ; S D=3.11)$. All of them were undergraduates at the University Jaume I (Spain), who voluntarily participated in exchange for course credit. Participants were right-handed native speakers of Spanish with no reported history of speech or hearing disorders.

\section{I.2 Materials}

The stimuli consisted of 80 Spanish spoken words and 80 spoken nonwords. Words were 40 early acquired words (AoA $M$ : 2.88years old) and 40 later acquired words (AoA $M$ : 9.28 years old). Words were selected from Álvarez and Cuetos (2007), a set of objective AoA norms collected from a pool of 760 children using a picture naming task. Objective AoA is a more accurate measure than the usual norms based on retrospective ratings made by adults. Early and later acquired words selected as experimental stimuli were matched on a number of relevant psycholinguistic variables obtained from the 
database EsPal (www.bcbl.eu/databases/espal/; Duchon et al., 2013): log (word frequency per million), number of letters, number of syllables, familiarity, imageability, concreteness, number of substitution neighbors, number of addition-letter neighbors, number of deletion-letter neighbors, and total number of neighbors (see Appendix 1). All nonwords were created by changing one phoneme from real and common Spanish words (not included in the experimental set).

As in González and McLennan's (2007) study, the stimuli (words and nonwords) were recorded in a sound-attenuated room by a male talker and digitized at a sampling rate of $44,100 \mathrm{~Hz}$ using a 16-bit analog-to-digital converter. Then they were down-sampled at $11,025 \mathrm{~Hz}$ and were low-pass filtered at $5.5 \mathrm{kHz} .{ }^{1}$ Stimuli were edited into individual wav sound files, converted to $\mathrm{mp} 3$ format, and stored for later playback. All audio files were equated in root mean square (RMS) amplitude. ${ }^{2}$

By contrast, a 700-ms audio file was created containing pink noise. The noise was low-pass filtered at $4 \mathrm{kHz}$ and digitized at a sampling rate of $8,000 \mathrm{~Hz}$ (16 bits). Finally, RMS amplitude was equated to the same level as the speech files, and converted to mp3 format. It has to be said that pink noise has a spectral frequency of $1 / \mathrm{f}$ and it was chosen because its spectral level decreases with increasing frequency, as occurs in speech signals, serving as an effective intelligibility masker being less annoying than white noise (González \& McLennan, 2007; González et al., 2010).

\section{I.3 Procedure}

The experiment was administered by means of the PsyToolkit software (Stoet, 2010, 2017) during two sessions separated by at least $60 \mathrm{~min}$. The stimuli were presented monaurally over headphones and simultaneously the pink noise in the opposite ear (as in González et al., 2010; González \& McLennan, 2007). The majority of auditory projections are contralateral (Kimura, 1967; Rosenzweig, 1951), and thus a stimulus presented to the right ear should be processed more quickly and more efficiently in the left hemisphere, and vice versa. Participants carried out a lexical decision task in which they had to decide as quickly and accurately as possible whether each speech stimulus they heard was a Spanish word or a nonword. They indicated their decision by pressing one of two keys on the computer keyboard (the "Y" key with the left forefinger, and the " $\mathrm{B}$ " key with the right forefinger; for half of the participants, the " $\mathrm{B}$ " indicated a word response, and for the other half this response was reversed).[AQ: 1]

Following a similar procedure as Bowers et al. (2013), each trial started with a central presentation of a white fixation cross for $400 \mathrm{~ms}$ on a black screen. After a 50 -ms blank interval, the fixation cross was displayed in red for $50 \mathrm{~ms}$. After another 50 -ms blank interval, the cross reappeared in white for $400 \mathrm{~ms}$. This sequence caused the appearance of a flickering fixation. Then, the participant was presented with a speech stimulus in an ear and simultaneously with the noise in the opposite ear. The presentation of noise in the opposite ear should increase competition between the hemispheres and augment the likelihood of observing hemispheric asymmetries (Behne et al., 2005, 2006; Kimura, 1961). RTs were recorded from the onset of the presentation of the stimulus to the keypress response. After the participant responded, the next trial was initiated $2 \mathrm{~s}$ later. If the maximum time window $(5 \mathrm{~s})$ expired with no response, the computer automatically recorded a missing response and presented the next trial.

Each speech stimulus was presented in both ears in different sessions in counterbalanced order. Within each session, no speech stimulus was repeated. The order of the two sessions was counterbalanced across the participants. In each session, each participant received a unique random ordering of trials. 


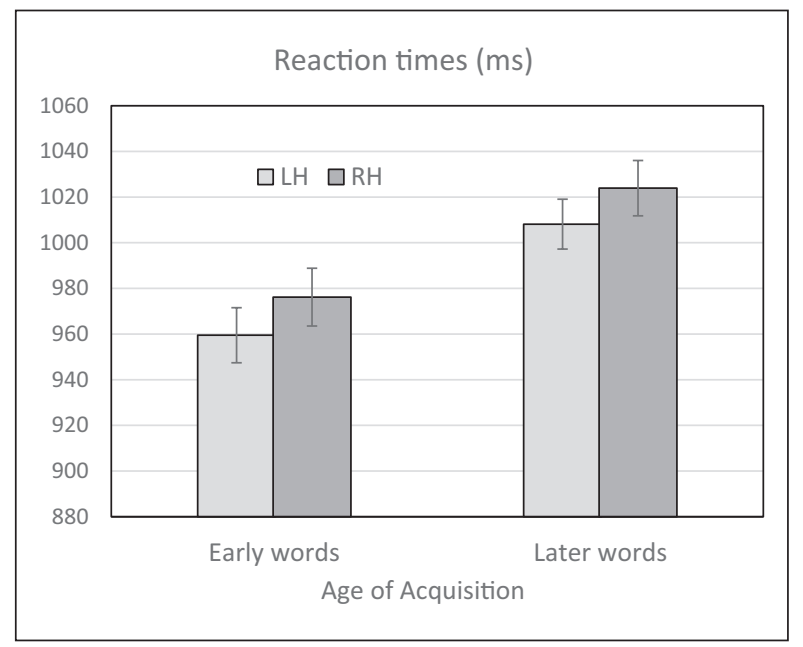

Figure I. Mean reaction times in milliseconds (ms) as a function of Age of Acquisition of spoken words for the left hemisphere $(\mathrm{LH})$ and right hemisphere $(\mathrm{RH})$. Error bars represent plus or minus one standard error of the mean.

\section{Results}

Accuracy to word stimuli was $90.4 \%$ overall. We considered RTs of correct responses to word stimuli. RTs smaller than $400 \mathrm{~ms}$ and larger than 3,000 ms were excluded from analysis. ${ }^{3}$ Mean RTs for early acquired words were the following: for the left hemisphere: $959 \mathrm{~ms}(S D=127), 95 \%$ confidence interval $(\mathrm{CI})=[936,983]$; for the right hemisphere: $976 \mathrm{~ms}(S D=133), 95 \% \mathrm{CI}=[951$, 1,001]. For later acquired words RTs were the following: for the left hemisphere: 1,008 ms $(S D=115), 95 \% \mathrm{CI}=[987,1030]$; for the right hemisphere: $1,024 \mathrm{~ms}(S D=128), 95 \% \mathrm{CI}=$ [1,000, 1,048] (see Figure 1).

A 2 (AoA, early vs. later words) $\times 2$ (hemisphere, left vs. right) ANOVA was carried out on RTs, and we made separate analyses across participants $\left(F_{1}\right)$ and items $\left(F_{2}\right)$. The analysis revealed a significant main effect of AoA through subjects, $F_{1}(1,110)=267.54, \mathrm{MS}_{\mathrm{e}}=963.60, p<.0001$, $\eta_{\mathrm{p}}^{2}=.709^{4}$; and through items, $F_{2}(1,78)=12.19, \mathrm{MS}_{\mathrm{e}}=9455.18, p<.001, \eta_{\mathrm{p}}^{2}=.135$, indicating that early acquired words showed smaller RTs $(M 968 \mathrm{~ms})$ than later acquired words $(1,016 \mathrm{~ms})$. Also, the analysis found a significant main effect of hemisphere through subjects, $F_{1}(1,110)=24.94$, $\mathrm{MS}_{\mathrm{e}}=1,172.21, p<.0001, \eta_{\mathrm{p}}^{2}=.185 ;$ and through items, $F_{2}(1,78)=25.91, \mathrm{MS}_{\mathrm{e}}=436.84$, $p<.0001, \eta_{\mathrm{p}}^{2}=.249$, indicating that the left hemisphere yielded smaller RTs (mean $984 \mathrm{~ms}$ ) than the right hemisphere $(1,000 \mathrm{~ms})$.

Crucially, the ANOVA did not reveal an AoA $\times$ Hemisphere interaction effect neither through subjects $\left(F_{1}<1\right)$ nor through items $\left(F_{2}<1\right)$, since the RT disparities between RH versus LH were not significantly different for early acquired words $(17 \mathrm{~ms})$ than for later acquired words $(16 \mathrm{~ms})$.

As a secondary dependent variable, we considered the accuracy of responses. Percentage of correct responses for early acquired words were the following: for the left hemisphere: $95.50 \%$ $(S D=0.57 \%), 95 \% \mathrm{CI}=[94.36 \%, 96.63 \%]$; for the right hemisphere: $95.22 \%(S D=0.56 \%), \mathrm{CI}=$ $[94.11 \%, 96.33 \%]$. For later acquired words percentages were the following: for the left hemisphere: $90.87 \%(S D=0.64 \%), 95 \% \mathrm{CI}=[89.60 \%, 92.14 \%]$; for the right hemisphere: $90.38 \%$ $(S D=0.64 \%), C I=[89.11 \%, 91.64 \%]($ see Figure 2$)$. 


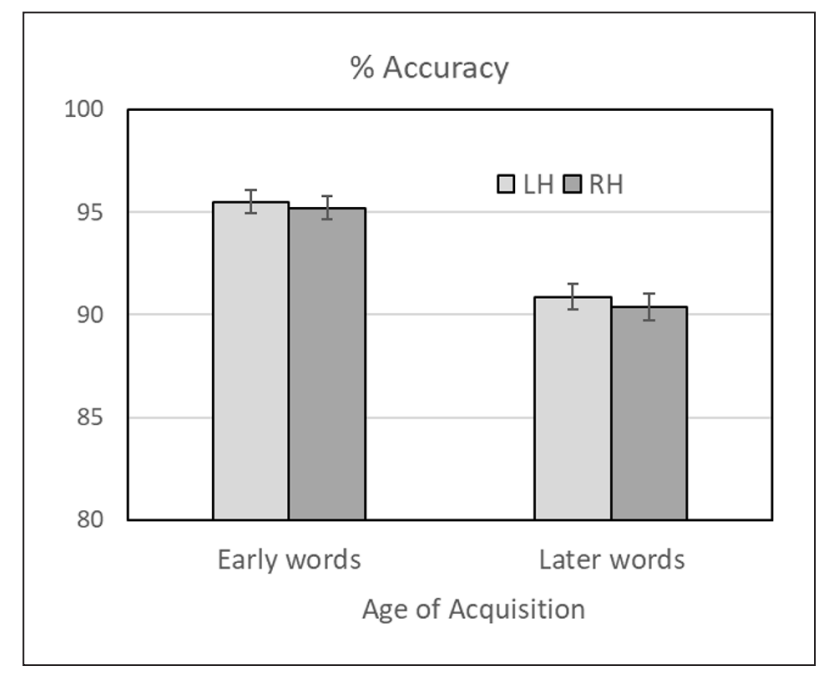

Figure 2. Percentages of accuracy of responses as a function of Age of Acquisition of spoken words for the left hemisphere $(\mathrm{LH})$ and right hemisphere $(\mathrm{RH})$. Error bars represent plus or minus one standard error of the mean.

A 2 (AoA, early vs. later words) $\times 2$ (hemisphere, left vs. right) ANOVA was performed on proportions of correct responses transformed into empirical logit values, ${ }^{5}$ and we made separate analyses across participants $\left(F_{1}\right)$ and items $\left(F_{2}\right)$. The analysis found a significant main effect of AoA through subjects, $F_{1}(1,110)=147.675, \mathrm{MS}_{\mathrm{e}}=0.624, p<.0001, \eta_{\mathrm{p}}^{2}=.573$; and through items, $F_{2}(1,78)=7.50, \mathrm{MS}_{\mathrm{e}}=1.610, p<.01, \eta_{\mathrm{p}}^{2}=.097$, indicating that early acquired words obtained larger accuracy ( $M 95.36 \%)$ than later acquired words $(90.62 \%)$. However, the analysis did not find a significant main effect of hemisphere through subjects $\left(F_{1}<1\right)$ nor through items $\left(F_{2}<1\right)$. Importantly, the ANOVA did not reveal an AoA $\times$ Hemisphere interaction effect neither through subjects $\left(F_{1}<1\right)$ nor through items $\left(F_{2}<1\right)$.

\section{Discussion}

According to the influential hypothesis of Gazzaniga (1974), words that are learned very early, that is, in the first years of childhood, would have a bilateral brain representation (or at least, a representation that is more bilateral than that of the words learned later). This is because the corpus callosum that connects the two hemispheres has not matured and myelinated enough to effectively connect the two brain halves; thus, functionally, the child has a split brain in which both hemispheres act parallelly. In contrast, words learned later, that is, once the corpus callosum had matured and language has specialized in the dominant hemisphere (left for most people), would have a more asymmetric representation, focused on the left hemisphere.

The empirical testing of the hypothesis, however, with studies conducted on adult participants has not proved this right. A majority of experiments has found that both the words acquired early in life as well as those acquired later have a lateralized representation in the left hemisphere to the same degree (Beaton et al., 2007; Boles et al., 1982; Ellis \& Young, 1977; Young et al., 1982). A recent study based on RTs Bowers et al. (2013) surprisingly found that early words were not only not represented bilaterally in the brain but were also processed slightly better (i.e., had shorter RTs) in the right hemisphere. Their results, the authors indicated, were in line with the researchers who 
suggest that the right hemisphere may play a relevant role in the first 3 years of life (for a review, see Dehaene-Lambertz et al., 2006).

All these studies were performed with stimuli consisting of written words. However, if we want to compare the processing of words acquired very early, that is, before the child can read, versus words acquired later, it seems more logical to use spoken words as experimental stimuli. In this way, the sensory modality of the stimuli would coincide with the modality in which they were learned in childhood. Young and Ellis (1980) carried out an initial study with spoken words, but unfortunately, they did not use online measures (RTs) on lexical processing. These authors also found no differences in the degree of lateralization between early and late words.

In the present study, based on the RTs in a lexical decision task, we used spoken words classified according to an objective measure of the AoA and selected according to two very distant mean ages (2.88 vs. 9.28 years old). Our results are very clear: left-hemisphere processing superiority (shorter RTs) occurs in both early and later words, and also to the same degree. Overall, earlier words are processed more efficiently - they take less time to be recognized aurally - than late words (confirming a general superiority of early words found in the literature in a number of lexical tasks), but there is no interaction between the AoA and the cerebral hemisphere.

These results lead us to two questions: were the early words acquired laterally from the beginning, despite the fact that the corpus callosum was not fully mature? Or did the early words initially have a bilateral representation — or even a rightward representation — but later, given the plasticity of the brain and with use over the years, were lateralized toward the speech hemisphere? It should not be forgotten that all these lexical processing experiments are carried out on adult participants.

It seems that between 1 and 3 years of age, the blood flow at rest shows a right hemispheric predominance, principally owing to the activity in the posterior associative area, suggesting that the human right hemisphere develops its functions earlier than the left (Chiron et al., 1997). However, there is growing evidence that the structural and functional asymmetry of the language network appears in childhood much earlier than we had thought (Dehaene-Lambertz et al., 2006; Reynolds et al., 2019). Recent structural neuroimage studies have found that the macrostructural leftward asymmetry of the arcuate fasciculus, which connects the Broca and Wernicke areas, is fully established by 2 years of age (Reynolds et al., 2019). Behavioral and neuroimaging studies in infants show that a substantial number of language processes-phoneme identification, phonological awareness, or word segmentation-already occurs in the first year of life. These studies reveal a structural and functional leftward organization close to what is described in adults (Dehaene-Lambertz et al., 2006; Reynolds et al., 2019). Even the leftward asymmetry of functional language networks has been identified in neonates (Pena et al., 2003).

Therefore, it would not be improbable that the words learned early in infancy are processed by a brain already lateralized to the left in its linguistic functions - although the possibility that these words initially have a bilateral representation is not excluded, and that, later, with their continued use over the years, their orthographic and phonological representations are preferentially lateralized in the left hemisphere of adults.

To give adequate answers to the above questions, it would be necessary to carry out further lexical-processing experiments adapted to 2- to 3-year-old boys and girls to test the degree of lateralization of the words they know. Obtaining neuroimages or electrophysiological measurements (e.g., event-related potentials [ERP]) and analyzing the degree of lateralization of the brain response during word recognition would be of great help in this type of experiment. 


\section{Acknowledgements}

The authors thank Marc Brysbaert and an anonymous reviewer for their helpful and valuable comments received on an earlier version of this paper.

\section{Funding[GQ: 2]}

The author(s) disclosed receipt of the following financial support for the research, authorship, and/or publication of this article: This work was completed with resources provided by the University Jaume I (Spain).

\section{ORCID ID[GQ: 3]}

Julio González-Alvarez (iD https://orcid.org/0000-0002-0389-5263

\section{Notes}

1. Stimuli were down sampled at $11,025 \mathrm{~Hz}$ (cutoff $5.5 \mathrm{kHz}$ ) to lighten the operation of the PsyToolkit software (Stoet, 2010, 2017). However, our experience on previous spoken-word experiments showed that intelligibility (at $0-5,500 \mathrm{~Hz}$ ) is virtually complete.

2. Stimuli are available at this repository: https://github.com/jgajgajj/Age-of-Acquisition-and-SpokenWords-Examining-Hemispheric-Differences-in-Lexical-Processing-

3. According with our previous experience with Spanish spoken words, these conservative limits (400$3,000 \mathrm{~ms}$ ) are appropriate for a lexical decision task. Nevertheless, analysis with other criteria of exclusion, as 2.5 or $3 S D$ s beyond the grand mean, yielded the same pattern of results (González \& McLennan, 2007; McLennan \& González, 2012).

4. The effect size interpretations for $\eta_{\mathrm{p}}^{2}$ values are $.01=$ small, $.06=$ medium, and $.14=$ large.

5. Since most of the accuracy proportions had very high values close to the ceiling, it was more appropriate to transform the individual averages into empirical logit values. We used the formula $\ln , p /(1-p$, ), in which $p$ is the accuracy proportion. When the proportion was 1 , it was replaced by .99 to avoid division by 0 .

\section{References}

Álvarez, B., \& Cuetos, F. (2007). Objective age of acquisition norms for a set of 328 words in Spanish. Behavior Research Methods, 39(3), 377-383.

Beaton, A. A., Suller, S., \& Workman, L. (2007). Visual laterality effects in readers of a deep and a shallow orthography. Laterality, 12(3), 199-215.

Behne, N., Scheich, H., \& Brechmann, A. (2005). Contralateral white noise selectively changes right human auditory cortex activity caused by a FM-direction task. Journal of Neurophysiology, 93(1), 414-423.

Behne, N., Wendt, B., Scheich, H., \& Brechmann, A. (2006). Contralateral white noise selectively changes left human auditory cortex activity in a lexical decision task. Journal of Neurophysiology, 95(4), 26302637.

Boles, D. B., Rogers, S., \& Wymer, W. (1982). Age of acquisition and visual field asymmetry in word recognition. Perception \& Psychophysics, 32(5), 486-490.

Bowers, J. M., Bradley, K. I., \& Kennison, S. M. (2013). Hemispheric differences in the processing of words learned early versus later in childhood. The Journal of General Psychology, 140(3), 174-186.

Brysbaert, M., \& Ellis, A. W. (2016). Aphasia and age of acquisition: Are early-learned words more resilient? Aphasiology, 30(11), 1240-1263.

Chiron, C., Jambaque, I., Nabbout, R., Lounes, R., Syrota, A., \& Dulac, O. (1997). The right brain hemisphere is dominant in human infants. Brain: A Journal of Neurology, 120(6), 1057-1065.

Cortese, M. J., \& Khanna, M. M. (2007). Age of acquisition predicts naming and lexical-decision performance above and beyond 22 other predictor variables: An analysis of 2,342 words. Quarterly Journal of Experimental Psychology, 60(8), 1072-1082. 
Dehaene-Lambertz, G., Hertz-Pannier, L., \& Dubois, J. (2006). Nature and nurture in language acquisition: Anatomical and functional brain-imaging studies in infants. Trends in Neurosciences, 29(7), 367-373.

Duchon, A., Perea, M., Sebastián-Gallés, N., Martí, A., \& Carreiras, M. (2013). EsPal: One-stop shopping for Spanish word properties. Behavior Research Methods, 45(4), 1246-1258.

Ellis, H. D., \& Young, A. W. (1977). Age-of-acquisition and recognition of nouns presented in the left and right visual fields: A failed hypothesis. Neuropsychologia, 15(6), 825-828.

Gazzaniga, M. S. (1974). Cerebral dominance viewed as a decision system. Hemisphere function in the human brain. In S. Dimond \& J. Beaumont (Eds.), Hemisphere function in the human brain (pp. 367-382). John Wiley \& Sons.

Gilhooly, K. J., \& Logie, R. H. (1980). Age-of-acquisition, imagery, concreteness, familiarity, and ambiguity measures for 1,944 words. Behavior Research Methods \& Instrumentation, 12(4), 395-427.

González, J., Cervera-Crespo, T., \& McLennan, C. T. (2010). Hemispheric differences in specificity effects in talker identification. Attention, Perception, \& Psychophysics, 72(8), 2265-2273.

González, J., \& McLennan, C. T. (2007). Hemispheric differences in indexical specificity effects in spoken word recognition. Journal of Experimental Psychology: Human Perception and Performance, 33(2), 410-424.

Johnston, R. A., \& Barry, C. (2006). Age of acquisition and lexical processing. Visual Cognition, 13(7-8), 789-845.

Kimura, D. (1961). Cerebral dominance and the perception of verbal stimuli. Canadian Journal of Psychology / Revue Canadienne de Psychologie, 15(3), 166-171.

McLennan, C. T., \& González, J. (2012). Examining talker effects in the perception of native-and foreignaccented speech. Attention, Perception, \& Psychophysics, 74(5), 824-830.

Nelson, K. (1973). Structure and strategy in learning to talk. Monographs of the Society for Research in Child Development, 38, 1-135.

Pena, M., Maki, A., Kovačić, D., Dehaene-Lambertz, G., Koizumi, H., Bouquet, F., \& Mehler, J. (2003). Sounds and silence: An optical topography study of language recognition at birth. Proceedings of the National Academy of Sciences, 100(20), 11702-11705.

Reynolds, J. E., Long, X., Grohs, M. N., Dewey, D., \& Lebel, C. (2019). Structural and functional asymmetry of the language network emerge in early childhood. Developmental Cognitive Neuroscience, 39, Article 100682 .

Rosenzweig, M. R. (1951). Representations of the two ears at the auditory cortex. American Journal of Physiology-Legacy Content, 167(1), 147-158.

Stoet, G. (2010). PsyToolkit - A software package for programming psychological experiments using Linux. Behavior Research Methods, 42(4), 1096-1104.

Stoet, G. (2017). PsyToolkit: A novel web-based method for running online questionnaires and reaction-time experiments. Teaching of Psychology, 44(1), 24-31.

Young, A. W., \& Bion, P. J. (1980). Hemifield differences for naming bilaterally presented nouns varying on age of acquisition. Perceptual and Motor Skills, 59, 366.

Young, A. W., Bion, P. J., \& Ellis, A. W. (1982). Age of reading acquisition does not affect visual hemifield asymmetries for naming imageable nouns. Cortex, 18(3), 477-482.

Young, A. W., \& Ellis, H. D. (1980). Ear asymmetry for the perception of monaurally presented words accompanied by binaural white noise. Neuropsychologia, 18(1), 107-110. 


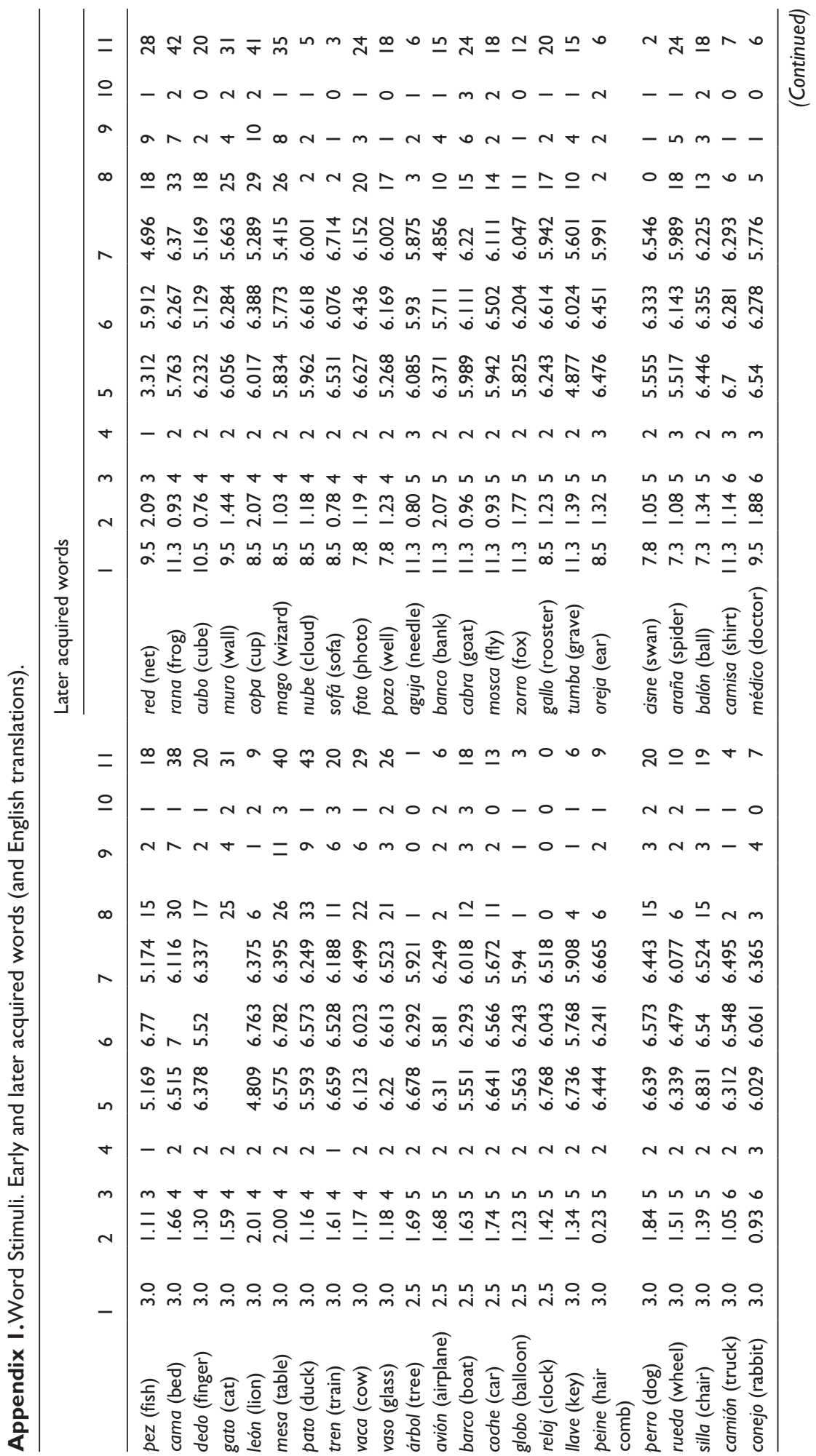




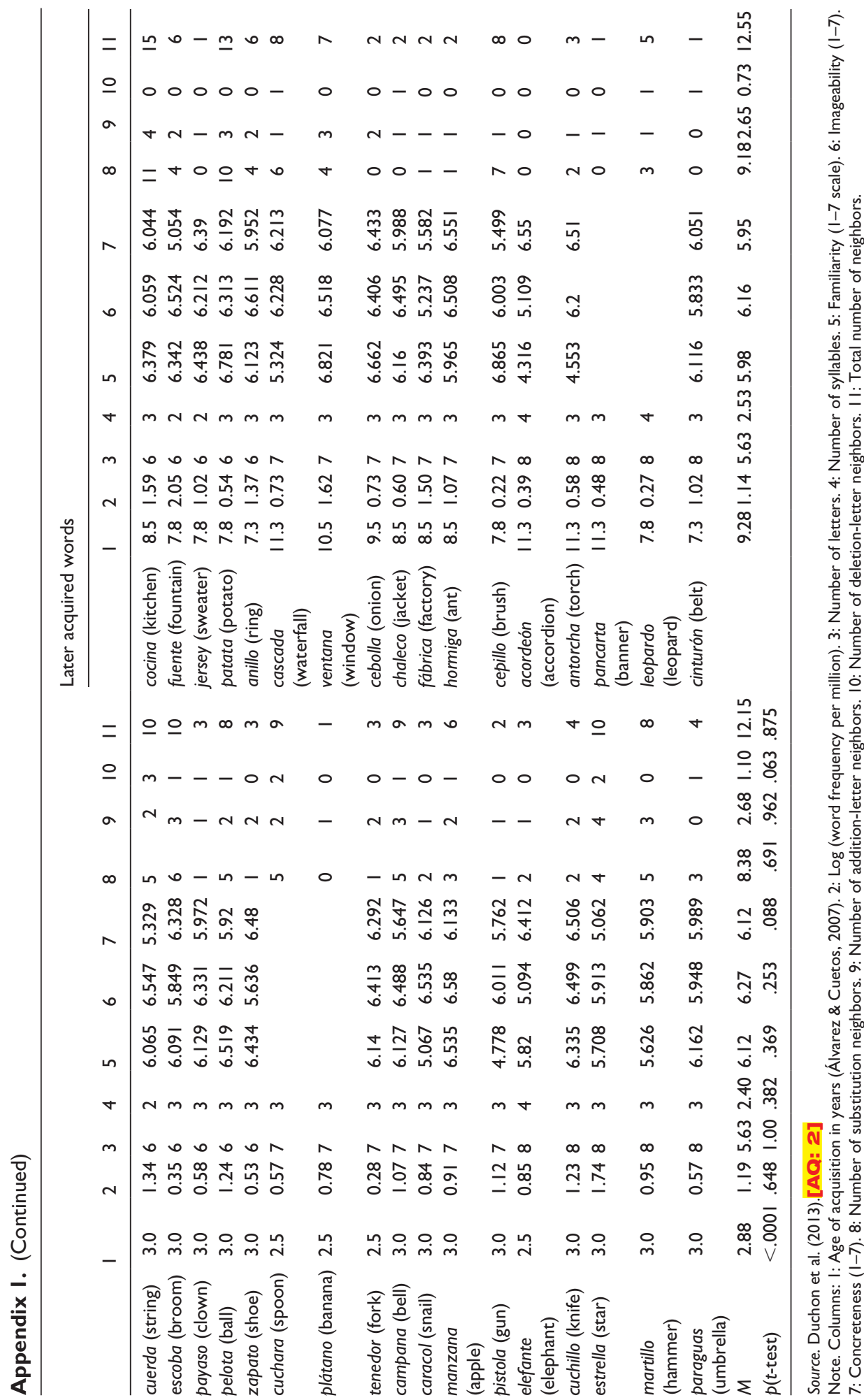

